\title{
Expansive homeomorphisms and indecomposability
}

by

Hisao Kato (Hiroshima)

Abstract. Suppose that $\mathbf{F}$ is a finite collection of graphs and a continuum $X$ is $\mathbf{F}$-like. If $X$ admits an expansive homeomorphism, then $X$ contains an indecomposable (nondegenerate) subcontinuum.

1. Introduction. A compact connected (nondegenerate) metric space is called a continuum. A homeomorphism $f: X \rightarrow X$ of a compact metric space $X$ is called expansive if there exists a constant $c>0$ (called an expansive constant for $f$ ) such that if $x, y \in X$ and $x \neq y$, then there is an integer $n=n(x, y) \in \mathbf{Z}$ such that

$$
d\left(f^{n}(x), f^{n}(y)\right)>c
$$

where $d$ is a metric of $X$. Expansiveness does not depend on the choice of metric of $X$. We are interested in the following problem [3]: What kinds of continua admit expansive homeomorphisms? We know that if a continuum $X$ is one-dimensional and admits an expansive homeomorphism, then $X$ is considerably complicated. In fact, we know that all known one-dimensional continua admitting expansive homeomorphisms contain indecomposable subcontinua which play important parts in the dynamics of the expansive homeomorphisms. For instance, Williams' examples are solenoids [26] and Plykin's examples are lakes of Wada [22] and [23], which are well-known indecomposable continua. Naturally, we are interested in the following problem (A): Is it true that if a one-dimensional continuum $X$ admits an expansive homeomorphism, then $X$ contains an indecomposable (nondegenerate) subcontinuum? Note that if $X$ is a continuum with $\operatorname{dim} X \geqslant 2$, then $X$ always contains a hereditarily indecomposable subcontinuum $Y$ with $\operatorname{dim} Y=\operatorname{dim} X-1$.

In this paper, we give a partial answer to problem (A). More precisely, the following theorem is proved: Suppose that $\mathbf{F}$ is a finite collection of graphs and a continuum $X$ is F-like. If $X$ admits an expansive homeomorphism, then $X$ contains an indecomposable

1980 Mathematics Subject Classification (1985 Revision): Primary 54F50; Secondary 54F20, $58 \mathrm{~F} 15$.

Words and phrases: expansive homeomorphism, $\Theta_{n}$-continuum, indecomposable, monotone decomposition, tree-like, crooked.

4 - Fundamenta Mathematicae 139 
subcontinuum. Note that for any continuum $X, \operatorname{dim} X \leqslant 1$ if and only if there is a countable collection $\mathbf{C}$ of graphs such that $X$ is $\mathbf{C}$-like. As a corollary, if $f: G \rightarrow G$ is an onto map of a graph $G$ such that the shift homeomorphism $f$ is expansive, then the inverse limit $(G, f)$ of $f$ contains an indecomposable subcontinuum.

We refer the readers to [1] for the general properties of expansive homeomorphisms.

2. Definitions and preliminaries. A compact connected polyhedron $P$ is called a graph if $\operatorname{dim} P=1$. Let $\mathbf{P}$ be a collection of graphs. A continuum $X$ is $\mathbf{P}$-like if for any $\varepsilon>0$ there is a map $f$ from $X$ to some member $P$ of $\mathbf{P}$ such that diam $f^{-1}(y)<\varepsilon$ for any $y \in f(X)$ ( $f$ is called an $\varepsilon$-map). In this paper, " $\varepsilon$-map" does not mean onto map. A compact metric space $X$ is tree-like if $X$ is a one-point set or $X$ is a T-like continuum, where $\mathbf{T}=\{$ all trees (= all graphs without simple closed curves) $\}$. Note that a continuum $X$ is $\mathbf{F}$-like for some finite collection $\mathbf{F}$ of graphs if and only if $X$ is $\{G\}$-like for some graph $G$ contained in $\mathbf{F}$.

An onto map $f: X \rightarrow Y$ is monotone if for any $y \in Y, f^{-1}(y)$ is connected. A continuum $X$ is called a $\Theta_{n}$-continuum if for any subcontinuum $Y$ of $X$ the complement $X-Y$ of $Y$ in $X$ has at most $n$ components. We can easily see that if a continuum $X$ is $\mathbf{F}$-like where $\mathbf{F}$ is a finite collection of graphs, then there is a natural number $n$ such that if $A$ and $B_{1}, \ldots, B_{m}$ are any subcontinua of $X$ satisfying

(1) $A \cap B_{i} \neq \varnothing$ for $i=1, \ldots, m$,

(2) $B_{i}$ is not contained in $A \cup\left(\bigcup_{j=1, j \neq i}^{m} B_{j}\right)$ for $i=1, \ldots, m$,

then $m \leqslant n$. By this fact, the following proposition is easily proved.

(2.1) Proposition. Let $\mathbf{F}$ be a finite collection of graphs. If a continuum $X$ is F-like, then there is a natural number $n$ such that $X$ is a $\Theta_{n}$-continuum.

A continuum $X$ is decomposable if $X$ is the union of two subcontinua different from $X$. A continuum $X$ is indecomposable if $X$ is not decomposable. A continuum $X$ is hereditarily decomposable (resp. hereditarily indecomposable) if each nondegenerate subcontinuum of $X$ is decomposable (resp. indecomposable).

We refer the readers to [17] for the properties of decomposable and indecomposable continua.

The following theorem obtained by Grace and Vought is very useful $([5$, Theorem 2] and [25, Theorem 7]).

(2.2) THEOREM. Let $X$ be a hereditarily decomposable $\Theta_{n}$-continuum. Then $X$ admits an upper semicontinuous monotone decomposition $\mathscr{D}$ such that $X / \mathscr{D}$ is a (nondegenerate) graph which is a $\Theta_{n}$-continuum. Furthermore, $\mathscr{D}=\left\{T^{2 n}(x) \mid x \in X\right\}$, where for any subcontinuum $A$ of $X, T(A)=A \cup\{x \in X-A \mid$ there is no subcontinuum $H$ of $X$ such that $x \in \operatorname{Int}(H) \subset H \subset X-A\}$, and $T^{0}(A)=A, T^{k}(A)=T\left(T^{k-1}(A)\right)$ for $k \geqslant 1$.

Note that each homeomorphism $f: X \rightarrow X$ satisfies $f(T(A))=T(f(A))$ for any subcontinuum $A$ of $X$.

3. Main theorem. In this section, we prove the following main theorem of this paper.
(3.1) THEOREM. Suppose that $\mathbf{F}$ is a finite collection of graphs and a continuum $X$ is F-like. If $X$ admits an expansive homeomorphism, then $X$ contains an indecomposable (nondegenerate) subcontinuum.

To prove (3.1), we need the following results and notations.

(3.2) Propositron ([24, (2.2)]). If $f: X \rightarrow X$ is an expansive homeomorphism of a compact metric space $X$, then for any integer $n \in \mathbf{Z}(n \neq 0), f^{n}: X \rightarrow X$ is also expansive.

(3.3) Lemma ([12, Lemma 2.2]). Let $f: X \rightarrow X$ be an expansive homeomorphism of a compact metric space $X$. Then there exists $\delta>0$ such that for each nondegenerate subcontinuum $A$ of $X$, there exists an integer $n_{0}>0$ satisfying one of the following conditions:

(*) $\operatorname{diam} f^{\prime \prime}(A) \geqslant \delta$ for all $n \geqslant n_{0}$, or

(**) $\operatorname{diam} f^{-n}(A) \geqslant \delta$ for all $n \geqslant n_{0}$.

In particular, if $A$ is a nondegenerate subcontinuum of $X$ such that $f^{N}(A)=A$ for some integer $N \neq 0$, then $\operatorname{diam} A \geqslant \delta$.

By a refinement of a finite collection $\mathscr{U}$ of subsets of a space $X$ we mean, as usual, any finite collection of subsets of $X$ whose elements are contained in elements of $\mathscr{U}$. Let $C_{1}, \ldots, C_{m}$ be a sequence of subsets of a space $X$. It is said to be a chain, and is denoted by $\left[C_{1}, \ldots, C_{m}\right]$, provided that $C_{i} \cap C_{j} \neq \varnothing$ if and only if $|i-j| \leqslant 1$ for each $1 \leqslant i, j \leqslant m$. A chain $\left[C_{1}, \ldots, C_{m}\right]$ is said to be an $\eta$-chain $(\eta>0)$ if $\operatorname{diam} C_{i}<\eta$ for each $i$. Let $\left[V_{1}, \ldots, V_{m}\right]$ be a chain such that $\mathscr{V}=\left\{V_{i} \mid 1 \leqslant i \leqslant m\right\}$ is a refinement of a finite open cover $\mathscr{U}$ of a space $X$. Let $U_{1}, U_{2} \in \mathscr{U}$. Then the chain $\left[V_{1}, \ldots, V_{m}\right]$ is said to be crooked between $U_{1}$ and $U_{2}$ if there are $1 \leqslant i(1)<i(2)<i(3)<i(4) \leqslant m$ such that $V_{i(1)} \subset U_{1}$, $V_{l(2)} \subset U_{2}, V_{i(3)} \subset U_{1}$ and $V_{i(4)} \subset U_{2}$. A chain $\left[V_{1}, \ldots, V_{m}\right]$ is said to be a chain from $x$ to $y(x, y \in X)$ if $x \in V_{1}$ and $y \in V_{m}$.

(3.4) LEMMA $([13,(4.6)])$. Let $f: X \rightarrow X$ be an expansive homeomorphism of a continuum $X$. Then there exists $\delta>0$ such that if $x, y \in X, x \neq y$, and $\mathscr{U}$ is any finite open cover of $X$, then there is an integer $N>0$ and $\eta>0$ such that if $\left[V_{1}, \ldots, V_{m}\right]$ is any $\eta$-chain from $x$ to $y$, then either the chain $\left[f^{N}\left(V_{1}\right), \ldots, f^{N}\left(V_{m}\right)\right]$ or the chain $\left[f^{-N}\left(V_{1}\right), \ldots, f^{-N}\left(V_{m}\right)\right]$ is a refinement of $\mathscr{U}$, and is crooked between $U_{s}$ and $U_{t}$, where $U_{s}, U_{t} \in \mathscr{U}$ and $d\left(U_{s}, U_{t}\right) \geqslant \delta-2 \operatorname{mesh}(\mathscr{U})$.

(3.5) Lemma (cf. $[13,(5.1)]$ ). Suppose that $f: X \rightarrow X$ is a homeomorphism of a continuum $X$ and $h: Z \rightarrow Z$ is a homeomorphism of a continuum $Z$. Also, suppose that $F: X \rightarrow Z$ is an onto map from $X$ onto $Z$ such that $F^{-1}(z)$ is hereditarily decomposable and tree-like for any $z \in Z$, and for some $z \in Z, F^{-1}(z)$ is nondegenerate (i.e., $F$ is not a homeomorphism). If the diagram

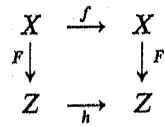

is commutative, then $f$ is not expansive.

Proof. Consider the set

$$
H=\{(x, y) \in X \times X \mid F(x)=F(y)\} .
$$


For any cover $\mathscr{U}$ of $X$, put $\overline{\mathscr{U}}=\{\mathrm{Cl}(U) \mid U \in \mathscr{U}\}$. For any subset $M$ of $H$, define $M^{f}=\left\{(x, y) \in H \mid\right.$ for any $\gamma>0$ and any finite open cover $\mathcal{U}$ of $X$, there exists $\left(x^{\prime}, y^{\prime}\right) \in M$ such that $x^{\prime} \neq y^{\prime}$ and there exists a finite open cover $\mathscr{V}$ of $X$ with mesh $(\mathscr{V})<\gamma$ such that $\overline{\mathscr{V}}$ is a refinement of $\mathscr{U}, \overline{\mathscr{V}}\left(F\left(x^{\prime}\right)\right)=\left\{\mathrm{Cl}(V) \mid V \in \mathscr{W}, V \cap F^{-1}\left(F\left(x^{\prime}\right)\right) \neq \varnothing\right\}$ is a refinement of $\mathscr{U}(F(x))=\left\{U \in \mathscr{U} \mid U \cap F^{-1}(F(x)) \neq \varnothing\right\}$, the nerve $N\left(\mathscr{V}\left(F\left(x^{\prime}\right)\right)\right)$ is a tree, and a chain $\left[V_{1}, \ldots, V_{m}\right]$ from $x^{\prime}$ to $y^{\prime}$ in $\mathscr{V}\left(F\left(x^{\prime}\right)\right)$ is crooked between $U_{x}, U_{y}$, where $U_{x}$ and $U_{y}$ are elements of $\mathscr{U}$ such that $x \in U_{x}$ and $\left.y \in U_{y}\right\}$.

Then $M^{f}$ is closed in $H$ and $M^{f} \supset\left(M^{f}\right)^{f}$. For any ordinal numbers, define $M_{1}=M^{f}, M_{\alpha+1}=\left(M_{\alpha}\right)^{f}$, and $M_{\lambda}=\bigcap_{\alpha<\lambda} M_{\alpha}$, where $\lambda$ is a limit ordinal.

First, we shall prove the following.

Claim (I). There is a countable ordinal $\alpha$ such that $M_{\alpha}=\varnothing$.

Proof. Note that $H$ is separable. Since $M_{\alpha}$ is closed in $H$ and $M_{\alpha} \supset M_{\beta}$ for $\alpha \leqslant \beta$, there is a countable ordinal $\alpha$ such that $M_{\alpha}=M_{\beta}$ for all $\alpha \leqslant \beta$. In particular, $\left(M_{\alpha}\right)^{f}=M_{\alpha}$. We shall show that $M_{\alpha}=\varnothing$. Suppose, on the contrary, that $M_{\alpha} \neq \varnothing$. Choose $\left(x_{1}, y_{1}\right) \in M_{\alpha}$. By the definition of $M^{f}$, we may assume that $x_{1} \neq y_{1}$. Since $F^{-1}(z)$ is tree-like for any $z \in Z$, there is a finite open cover $\mathscr{U}_{1}$ of $X$ such that

a(1) $\operatorname{mesh}\left(\mathscr{U}_{1}\right)<\min \left\{1 / 2, d\left(x_{1}, y_{1}\right) / 3\right\}$, and

$\mathrm{b}(1)$ the nerve $N\left(\mathscr{U}_{1}\left(F\left(x_{1}\right)\right)\right)$ is a tree, where $\mathscr{U}_{1}\left(F\left(x_{1}\right)\right)=\left\{U \in \mathscr{U}_{1} \mid U \cap\right.$ $\left.F^{-1}\left(F\left(x_{1}\right)\right) \neq \varnothing\right\}$.

By induction, we can choose $\left(x_{i}, y_{i}\right) \in\left(M_{\alpha}\right)^{f}=M_{\alpha}(i=1,2, \ldots)$ and a finite open cover $\mathscr{U}_{i}$ of $X$ such that

a $(i) \operatorname{mesh}\left(\mathscr{U}_{i}\right)<1 / 2^{i}, \overline{\mathscr{U}}_{i+1}$ is a refinement of $\mathscr{U}_{i}$ and $\overline{\mathscr{U}}_{i+1}\left(F\left(x_{i+1}\right)\right)$ is a refinement of $\mathscr{U}_{i}\left(F\left(x_{i}\right)\right.$ ),

$\mathrm{b}(i)$ the nerve $N\left(\mathscr{U}_{i}\left(F\left(x_{i}\right)\right)\right)$ is a tree, and

$\mathrm{c}(i)$ a chain $\left[U_{1}^{i+1}, \ldots, U_{m(i+1)}^{i+1}\right]$ from $x_{i+1}$ to $y_{i+1}$ in $\mathscr{U}_{i+1}\left(F\left(x_{i+1}\right)\right)$ is crooked between $U_{x_{i}}^{i}$ and $U_{y_{i}}^{i}$, where $x_{i} \in U_{x_{i}}^{i} \in \mathscr{U}_{i}$ and $y_{i} \in U_{y_{i}}^{i} \in \mathscr{U}_{i}$.

By $\mathrm{b}(i)$ and $\mathrm{c}(i)$, for each $i=1,2, \ldots$, we can choose a subchain $\left[U_{x_{1}}^{l}, \ldots, U_{y_{1}}^{l}\right]$ of $\left[U_{1}^{i}, \ldots, U_{m(i)}^{i}\right]$ such that

(1) $U_{x_{1}}^{1} \supset \mathrm{Cl}\left(U_{x_{1}}^{2}\right) \supset U_{x_{1}}^{2} \supset \mathrm{Cl}\left(U_{x_{1}}^{3}\right) \supset \ldots$

(2) $U_{y_{1}}^{1} \supset \mathrm{Cl}\left(U_{y_{1}}^{2}\right) \supset U_{y_{1}}^{2} \supset \mathrm{Cl}\left(U_{y_{1}}^{3}\right) \supset \ldots$, and

(3) $\left[U_{x_{1}}^{i+1}, \ldots, U_{y_{1}}^{i+1}\right]$ is crooked between $U_{x_{1}}^{i}$ and $U_{y_{1}}^{i}$.

By a $(i), \lim _{i \rightarrow \infty} \mathrm{Cl}\left(U_{x_{1}}^{i}\right)=x$ and $\lim _{i \rightarrow \infty} \mathrm{Cl}\left(U_{y_{1}}^{i}\right)=y$. Note that $x \neq y$ and $F(x)=F(y)$. Also, $F^{-1}(z)$ is tree-like for each $z \in Z$. Put $Y=\bigcap_{i=1}^{\infty} G_{l}$, where $G_{i}=\bigcup\left\{\mathrm{Cl}(U) \mid U \in \mathscr{U}_{i}\left(F\left(x_{i}\right)\right)\right\}$. By a $(i)$ and $\mathrm{b}(i), Y$ is a continuum such that $Y \subset F^{-1}(F(x))$ and $x, y \in Y$. Let $K$ be the irreducible subcontinuum between $x$ and $y$ in $F^{-1}(F(x))$. Since $F^{-1}(F(x))$ is tree-like, we see that $K \subset Y$. Thus $K$ is nondegenerate.

We shall show that $K$ is indecomposable. Suppose, on the contrary, that there are two proper subcontinua $A$ and $B$ of $K$ such that $K=A \cup B$. Since $K$ is irreducible between $x$ and $y$ in $Y$, we may assume that $x \in A-B$ and $y \in B-A$. By a(i), we can choose $i$ such that $\mathrm{Cl}\left(U_{x_{1}}^{i}\right) \cap B=\varnothing$ and $\mathrm{Cl}\left(U_{y_{1}}^{i}\right) \cap A=\varnothing$. Consider the cover $\mathscr{U}_{i+1}\left(F\left(x_{i+1}\right)\right)$ of $F^{-1}\left(F\left(x_{i+1}\right)\right)$. Note that the nerve $N\left(\mathscr{U}_{i+1}\left(F\left(x_{i+1}\right)\right)\right)$ is a tree and
$\mathscr{U}_{i+1}\left(F\left(x_{i+1}\right)\right)$ is a cover of $K$. By (3), there are two subchains $\left[U_{x_{1}}^{i+1}, \ldots, U_{p}^{i+1}\right]$ and $\left[U_{q}^{i+1}, \ldots, U_{y_{1}}^{i+1}\right]$ of $\left[U_{x_{1}}^{i+1}, \ldots, U_{y_{1}}^{i+1}\right]$ such that $p<q$ and $U_{p}^{i+1} \subset U_{y_{1}}^{i}, U_{q}^{i+1} \subset U_{x_{1}}^{i}$. Then $A \cap U_{x_{1}}^{i+1} \neq \varnothing, \quad B \cap U_{y_{1}}^{i+1} \neq \varnothing, \quad A \cap U_{p}^{i+1}=\varnothing \quad$ and $B \cap U_{q}^{i+1}=\varnothing$. Since $N\left(\mathcal{U}_{i+1}\left(F\left(x_{i+1}\right)\right)\right)$ is a tree, we see that $A \cap B=\varnothing$. This is a contradiction.

Hence $K$ is indecomposable. Since $F^{-1}(F(x))$ is hereditarily decomposable, this is a contradiction. Hence, Claim $(\mathrm{I})$ is true.

Next, we shall prove the following.

Claim (II). If $f: X \rightarrow X$ is an expansive homeomorphism, then $M_{\alpha} \neq \varnothing$ for any countable ordinal $\alpha$, where $M=H$.

Proof. Let $\delta>0$ be a positive number satisfying the conditons as in (3.4). Choose $z_{0} \in Z$ such that $F^{-1}\left(z_{0}\right)$ is nondegenerate. Let $x_{0}, y_{0} \in F^{-1}\left(z_{0}\right)$ with $x_{0} \neq y_{0}$. Choose a sequence $\mathscr{U}_{1}, \mathscr{U}_{2}, \ldots$ of finite open coverings of $X$ such that

(1) $\overline{\mathscr{U}}_{i+1}$ is a refinement of $\mathscr{U}_{l}$, and

(2) $\operatorname{mesh}\left(\mathscr{U}_{i}\right)<1 / 2^{i}$ for each $i$.

According to (3.4), there is a sequence $\mathscr{V}_{1}, \mathscr{V}_{2}, \ldots$ of finite open coverings of $X$ such that for some integer $N(i) \in \mathbf{Z}$,

(3) $f^{N(i)}\left(\overline{\mathscr{V}}_{i}\right)$ is a refinement of $\mathscr{U}_{i}$,

(4) $f^{N(i)}\left(\mathscr{V}_{i}\left(F\left(x_{0}\right)\right)\right)$ is a refinement of $\mathscr{U}_{i}\left(h^{N(i)}\left(F\left(x_{0}\right)\right)\right)$ and $N\left(\mathscr{V}_{i}\left(F\left(x_{0}\right)\right)\right)$ is a tree,

(5) if $\left[V_{1}, \ldots, V_{m(i)}\right]$ is a chain from $x_{0}$ to $y_{0}$ in $\mathscr{V}_{i}\left(F\left(x_{0}\right)\right)$, then $\left[f^{N(i)}\left(V_{1}\right), \ldots, f^{N(i)}\left(V_{m(i)}\right)\right]$ is crooked between $U_{1}^{i}$ and $U_{2}^{i}$, where $U_{1}^{i}$ and $U_{2}^{i}$ are elements of $\mathscr{U}_{i}\left(h^{N(i)}\left(F\left(x_{0}\right)\right)\right)$ and $d\left(U_{1}^{l}, U_{2}^{l}\right) \geqslant \delta-2 \operatorname{mesh}\left(\mathscr{U}_{i}\right)$ for each $i$.

Since $X$ is compact, we may assume that $\lim _{i \rightarrow \infty} \mathrm{Cl}\left(U_{1}^{i}\right)=x_{1}$ and $\lim _{i \rightarrow \infty} \mathrm{Cl}\left(U_{2}^{i}\right)$ $=y_{1}$. We will show that $\left(x_{1}, y_{1}\right) \in M_{1}$ and $d\left(x_{1}, y_{1}\right) \geqslant \delta$. Clearly, $\left(x_{1}, y_{1}\right) \in M(=H)$ and $d\left(x_{1}, y_{1}\right) \geqslant \delta$ (see (5)). Let $\mathscr{U}$ be any finite open cover of $X$ and let $\gamma>0$. By the constructions of $x_{1}$ and $y_{1}$, there is $i$ such that $\mathrm{Cl}\left(U_{1}^{i}\right) \subset U_{x_{1}}, \mathrm{Cl}\left(U_{2}^{i}\right) \subset U_{y_{1}}$ and $\overline{\mathscr{U}}_{i}$ is a refinement of $\mathscr{U}$ with $\operatorname{mesh}\left(\mathscr{U}_{i}\right)<\gamma$, where $U_{x_{1}}$ (resp. $U_{y_{1}}$ ) is an element of $\mathscr{U}$ containing $x_{1}$ (resp. $y_{1}$ ). We may assume that $f^{N(i)}\left(\overline{\mathscr{V}}_{i}\left(F\left(x_{0}\right)\right)\right)$ is a refinement of $U\left(F\left(x_{1}\right)\right)$, because $F^{-1}$ is upper semicontinuous. By $(4)$ and $(5),\left(f^{N(i)}\left(x_{0}\right), f^{N(i)}\left(y_{0}\right)\right)$ and $f^{N(i)}\left(\mathscr{V}_{i}\right)$ satisfy the conditions of the definition of $M^{f}$. Hence $\left(x_{1}, y_{1}\right) \in M^{f}=M_{1}$.

For a countable ordinal $\lambda$, we assume that we have obtained $\left(x_{\alpha}, y_{\alpha}\right)$ in $M_{\alpha}$ for all $\alpha<\lambda$ such that $d\left(x_{\alpha}, y_{\alpha}\right) \geqslant \delta$. We will define $\left(x_{\lambda}, y_{\lambda}\right) \in M_{\lambda}$ recursively in the following way: Consider two cases.

(i) $\lambda=\alpha+1$. By an argument similar to the above one, we can obtain $\left(x_{\alpha+1}, y_{\alpha+1}\right) \in M_{\alpha+1}$ such that $d\left(x_{\alpha+1}, y_{\alpha+1}\right) \geqslant \delta$.

(ii) $\lambda$ is a limit ordinal. Take a sequence $\alpha_{1}<\alpha_{2}<\ldots$ of countable ordinals such that $\lim _{i \rightarrow \infty} \alpha_{i}=\lambda$. Note that $d\left(x_{\alpha_{1}}, y_{\alpha_{1}}\right) \geqslant \delta$. We may assume that $\left\{x_{\alpha_{i}}\right\}$ (resp. $\left\{y_{\alpha_{3}}\right\}$ ) converges to a point $x_{\lambda}$ (resp. $\left.y_{\lambda}\right)$ of $X$. Then $d\left(x_{\lambda}, y_{\lambda}\right) \geqslant \delta$ and $\left(x_{\lambda}, y_{\mu}\right) \in H$. Since $M_{\alpha}$ is closed in $H$ and $M_{\alpha} \supset M_{\beta}$ for $\alpha \leqslant \beta,\left(x_{\lambda}, y_{\lambda}\right) \in M_{\alpha_{i}}$ for all $i$. Hence

$$
\left(x_{\lambda}, y_{\lambda}\right) \in \bigcap_{i=1}^{\infty} M_{\alpha_{i}}=\bigcap_{\alpha<\lambda} M_{\alpha}=M_{\lambda} .
$$


Therefore $M_{\lambda} \neq \varnothing$ for any countable ordinal $\lambda$. Claim (II) contradicts Claim (I), which completes the proof.

Proof of (3.1). Let $f: X \rightarrow X$ be an expansive homeomorphism of $X$. Suppose, on the contrary, that $X$ is hereditarily decomposable. By (3.3), we can choose a minimal nondegenerate subcontinuum $X_{1}$ in the set $\{Z \mid Z$ is a nondegenerate subcontinuum of $X$ such that $f(Z)=Z\}$. Put $f_{1}=f \mid X_{1}: X_{1} \rightarrow X_{1}$. Note that $X_{1}$ is F-like. By (2.1), $X_{1}$ is a $\Theta_{n}$-continuum for some $n \geqslant 0$.

For any one-dimensional continuum $Y$, we define an index $I(Y)$ as follows: $I(Y) \leqslant m$ if for any $\varepsilon>0$ there is a finite open cover $\mathscr{U}$ of $Y$ such that the nerve $N(\mathscr{U})$ is a graph, mesh $(\mathscr{U})<\varepsilon$ and the number of all simple closed curves in $N(\mathscr{U})$ is equal to or less than $m$. When $I(Y) \leqslant m$ and $I(Y) \leqslant m-1$ is not true, we define $I(Y)=m$. Note that if $Z$ is any subcontinuum of $Y$, then $I(Z) \leqslant I(Y)$.

Since $\mathbf{F}$ is a finite collection of graphs and $X_{1}$ is $\mathbf{F}$-like, there is a natural number $N$ such that $I\left(X_{1}\right)=N<\infty$. By (2.2), there are a monotone map $F_{1}: X_{1} \rightarrow G_{1}$ from $X_{1}$ onto a graph $G_{1}$ and a homeomorphism $h_{1}: G_{1} \rightarrow G_{1}$ making the following diagram $\left(\mathrm{C}_{1}\right)$ commute:

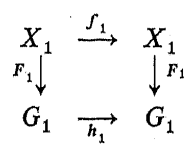

Since $h_{1}$ is not expansive (see [2], [7] and [8]), $F_{1}$ is not a homeomorphism.

Put $E_{1}=\left\{y \in G_{1} \mid F_{1}^{-1}(y)\right.$ is nondegenerate and not tree-like $\}$. Now, we shall prove that $E_{1}$ is a finite set, in fact the cardinal number $E_{1}^{\#}$ of $E_{1}$ is equal to or less than $N$. Suppose, on the contrary, that $E_{1}^{*} \geqslant N+1$. Choose $y_{1}, \ldots, y_{N+1}$ in $E_{1}$ such that $y_{i} \neq y_{j}(i \neq j)$ and $F_{1}^{-1}\left(y_{i}\right)$ is not tree-like for each $i$. Choose $\varepsilon$ such that $0<\varepsilon<\frac{1}{2} \min \left\{d\left(F_{1}^{-1}\left(y_{i}\right), F_{1}^{-1}\left(y_{j}\right)\right) \mid 1 \leqslant i \neq j \leqslant N+1\right\}$. Let $\varepsilon=\varepsilon_{1}>\varepsilon_{2}>\ldots$ be a sequence of positive numbers with $\lim _{i \rightarrow \infty} \varepsilon_{i}=0$. By the definition of $N$, we have finite open coverings $\mathscr{U}_{i}$ of $X_{1}$ such that $N\left(\mathscr{U}_{i}\right)$ is a graph, $\operatorname{mesh}\left(N\left(\mathscr{U}_{i}\right)\right)<\varepsilon_{i}$ for $i=1,2, \ldots$, and the number of simple closed curves in $N\left(\mathscr{U}_{i}\right)$ is $\leqslant N$ for each $i$. Hence we can choose $k_{0}$ and a subsequence $\left\{i_{j}\right\}$ of $\{1,2, \ldots\}$ such that $N\left(\mathscr{U}_{i_{j}}\left(F_{1}^{-1}\left(y_{k_{0}}\right)\right)\right)=N\left(\left\{U \in \mathscr{U}_{i_{j}}\right.\right.$ $\left.\left.U \cap F_{1}^{-1}\left(y_{k_{0}}\right) \neq \varnothing\right\}\right)$ is a tree, which implies that $F_{1}^{-1}\left(y_{k_{0}}\right)$ is tree-like. This is a contradiction. Therefore $E_{1}^{\#} \leqslant N$.

Since $f_{1}$ is a homeomorphism and by $\left(\mathrm{C}_{1}\right), h_{1}\left(E_{1}\right)=E_{1}$. By the definition of $X_{1}, h_{1}$ has no fixed points in $E_{1}$. If $E_{1} \neq \varnothing$, we can choose $y_{0}$ in $E_{1}$ such that $h_{1}^{n_{1}}\left(y_{0}\right)=y_{0}$ for some $n_{1} \geqslant 2$, and $h_{1}^{i}\left(y_{0}\right) \neq h_{1}^{j}\left(y_{0}\right)$ for $1 \leqslant i<j \leqslant n_{1}$. Put $X_{2}^{\prime}=F_{1}^{-1}\left(y_{0}\right)$ and $f_{2}^{\prime}=f_{1}^{n_{1}} \mid X_{2}^{\prime}: X_{2}^{\prime} \rightarrow X_{2}^{\prime}$. Note that $\left\{F_{1}^{-1}\left(y_{0}\right), F_{1}^{-1}\left(h_{1}\left(y_{0}\right)\right), \ldots, F_{1}^{-1}\left(h_{1}^{n_{1}-1}\left(y_{0}\right)\right)\right\}$ is a family of mutually disjoint continua which are not tree-like. Hence $I\left(X_{2}^{\prime}\right)<I\left(X_{1}\right)$. Take a minimal nondegenerate subcontinuum $X_{2}$ in the set $\{Z \mid Z$ is a nondegenerate subcontinuum of $X_{2}^{\prime}$ such that $\left.f_{2}^{\prime}(Z)=Z\right\}$ and put $f_{2}=f_{2}^{\prime} \mid X_{2}$. Note that $I\left(X_{2}\right)$ $\leqslant I\left(X_{2}^{\prime}\right)<I\left(X_{1}\right)=N$. By (2.2), there are a monotone map $F_{2}: X_{2} \rightarrow G_{2}$ from $X_{2}$ onto a graph $G_{2}$ and a homeomorphism $h_{2}: G_{2} \rightarrow G_{2}$ making the following diagram
$\left(\mathrm{C}_{2}\right)$ commute:

$$
\begin{array}{cccc}
X_{2} & \stackrel{f_{2}}{\rightarrow} & X_{2} \\
F_{2} \downarrow & & & \downarrow^{F_{2}} \\
G_{2} & \underset{h_{2}}{\rightarrow} & G_{2}
\end{array}
$$

Note that $f_{2}$ is also an expansive homeomorphism (see (3.2)). Put $E_{2}=\left\{y \in G_{2} \mid F_{2}^{-1}(y)\right.$ is nondegenerate and not tree-like\}. If $E_{2} \neq \varnothing$, we continue this process. Then we see that $\infty>N=I\left(X_{1}\right)>I\left(X_{2}\right)>I\left(X_{3}\right)>\ldots$ Note that $I(Y) \geqslant 0$ for any continuum $Y$, and $I(Y)=0$ if and only if $Y$ is tree-like. Clearly, we can reach the situation $E_{k}=\varnothing$, i.e., there are a nondegenerate subcontinuum $X_{k}$ of $X_{k-1}$, an expansive homeomorphism $f_{k}: X_{k} \rightarrow X_{k}$ of $X_{k}$ and a monotone map $F_{k}: X_{k} \rightarrow G_{k}$ from $X_{k}$ onto a graph $G_{k}$ such that $F_{k}^{-1}(y)$ is tree-like for any $y \in G_{k}$, and a homeomorphism $h_{k}$ of $G_{k}$ making the following diagram $\left(\mathrm{C}_{k}\right)$ commute:

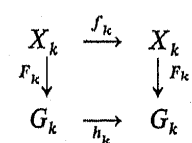

Since $h_{k}$ is not expansive, $F_{k}$ is not a homeomorphism. Note that $X_{k}$ is hereditarily decomposable.

By (3.5), this is a contradiction, which completes the proof.

Let $f: X \rightarrow X$ be a map of a compact metric space $X$ with a metric $d$ and let

$$
\left.(X, f)=\left\{\left(x_{i}\right)^{\infty}\right)_{0}^{\infty} \mid x_{i} \in X, f\left(x_{i+1}\right)=x_{i}, i \geqslant 0\right\} .
$$

Define a metric $\tilde{d}$ for $(X, f)$ by

$$
\tilde{d}(\tilde{x}, \tilde{y})=\sum_{i=0}^{\infty} d\left(x_{i}, y_{i}\right) / 2^{i}, \quad \text { for } \tilde{x}=\left(x_{i}\right)_{i=0}^{\infty}, \tilde{y}=\left(y_{i}\right)_{i=0}^{\infty} \in(X, f) .
$$

Then the space $(X, f)$ is called the inverse limit of the map $f$. Note that $(X, f)$ is a compact metric space. Also, define a map $\tilde{f}:(X, f) \rightarrow(X, f)$ by

$$
f\left(\left(x_{i}\right)_{i=0}^{\infty}\right)=\left(f\left(x_{i}\right)\right)_{i=0}^{\infty} \quad\left(=\left(f\left(x_{0}\right), x_{0}, x_{1}, \ldots,\right)\right) .
$$

Then $\tilde{f}$ is a homeomorphism and it is called the shift homeomorphism of $f$. Note that almost all examples of one-dimensional continua admitting expansive homeomorphisms are obtained as inverse limits of maps $f: G \rightarrow G$ of graphs whose shift homeomorphisms are expansive (see [11], [13], [14], [22], [23] and [26]).

(3.6) Corollary. Let $\mathbf{F}$ be a finite collection of graphs. If a continuum $X$ is homeomorphic to an inverse limit of an inverse sequence $\left\{G_{n}, f_{n, n+1}\right\}$ such that each $G_{n}$ is an element of $\mathbf{F}$, and $X$ admits an expansive homeomorphism, then $X$ contains an indecomposable subcontinuum. In particular, if $f: G \rightarrow G$ is a map of a graph $G$ such that $(G, f)$ is nondegenerate and the shift homeomorphism $\tilde{f}$ is expansive, then $(G, f)$ contains an indecomposable subcontinuum. 
(3.7) Remark. In the statement of (3.1) and (3.6), we can not conclude that $X$ itself is an indecomposable continuum.

The following problems remain open.

PROBLEM 1. Is it true that if a one-dimensional continuum $X$ admits an expansive homeomorphism, then $X$ contains an indecomposable subcontinuum?

PRoBlem 2. Is there a hereditarily indecomposable continuum admitting an expansive homeomorphism? In particular, does the pseudo-arc admit an expansive homeomorphism?

Problem 3. Is there a nonseparating plane continuum admitting an expansive homeomorphism? Moreover, is there a tree-like continuum admitting an expansive homeomorphism?

\section{References}

[1] N. A oki, Topological dynamics, in: Topics in General Topology, K. Morita and J. Nagata (eds.), Elsevier, 1989, 625-740.

[2] B. F. Bryant, Unstable self-homeomorphisms of a compact space, thesis, Vanderbilt University, 1954

[3] W. Gottschalk, Minimal sets; an introduction to topological dynamics, Bull. Amer. Math. Soc. 64 (1958), 336-351.

[4] W. Gottschalk and G. Hedlund, Topological dynamics, Amer. Math. Soc. Publ. Colloq. 36 (1955).

[5] E. E. Grace and E. J. Vought, Monotone decompositions of $\Theta_{n}$-continua, Trans. Amer. Math. Soc. 263 (1981), 261-270.

[6] K. Hiraide, Expansive homeomorphisms of compact surfaces are pseudo Anosov, Osaka J. Math. 27 (1990), 117-162.

[7] J. F. Jacobson and W. R. Utz, The nonexistence of expansive homeomorphisms of a closed 2-cell, Pacific J. Math. 10 (1960), 1319-1321.

[8] H. Kato, The nonexistence of expansive homeomorphisms of 1-dimensional compact ANRs, Proc. Amer. Math. Soc. 108 (1990), 267-269.

[9] -, The nonexistence of expansive homeomorphisms of Peano continua in the plane, Topology Appl. 34 (1990), 161-165.

[10] -, The nonexistence of expansive homeomorphisms of Suslinian continua, J. Math. Soc. Japan 42 (1990), 631-637.

[11] -, On expansiveness of shift homeomorphisms of inverse limits of graphs, Fund. Math. 137 (1991), 201-210.

[12] -, The nonexistence of expansive homeomorphisms of dendroids, ibid. 136 (1990), 37-43.

[13] -, Expansive homeomorphisms in continuum theory, Topology Appl., to appear.

[14] -, Embeddability into the plane and movability on inverse limits of graphs whose shift maps are expansive, ibid., to appear.

[15] H. Kato and K. Kaw a m u r a, A class of continua which admit no expansive homeomorphisms, Rocky Mountain J. Math., to appear.

[16] K. Kawamura, $A$ direct proof that Peano continuum with a free arc admits no expansive homeomorphisms, Tsukuba J. Math. 12 (1988), 521-524.

[17] K. Kuratowski, Topology, Vol. II, Academic Press, New York 1968.

[18] R. M añé, Expansive homeomorphisms and topological dimension, Trans. Amer. Math. Soc. 252 (1979), 313-319.
[19] T. O'Brien and W. Roddy, Each compact orientable surface of positive genus admits an expansive homeomorphism, Pacific J. Math. 35 (1970), 737-741.

[20] W. Reddy, The existence of expansive homeomorphisns of manifolds, Duke Math. J. 32 (1965), $627-632$.

[21] -, Expansive canonical coordinates are hyperbolic, Topology Appl. 15 (1983), 205-210.

[22] R. V. Ply kin, Sources and sinks of A-diffeomorphisms of surfaces, Math. USSR-Sb. 23 (1974) $233-253$.

[23] -, On the geometry of hyperbolic attractors of smooth cascades, Russian Math. Surveys 39 (1984), $85-131$

[24] W. R. Utz, Unstable homeomorphisms, Proc. Amer. Math. Soc. 1 (1950), 769-774.

[25] E. J. Vought, Monotone decompositions of continua, in: General Topology and Modern Analysis, L. F. McAuley and M. M. Rao (eds.), Academic Press, New York 1981, 105-113.

[26] R. F. Williams, A note on unstable homeomorphisms, Proc. Amer. Math. Soc. 6 (1955), 308 309 .

FACULTY OF INTEGRATEID ARTS ANID SCIENCES MA UNIVERSITY

Hiroshima 730, Japan
Received 14 May 1990;

in revised form 5 November 1990 